ARTICLE

\title{
High damage tolerance of electrochemically lithiated silicon
}

Xueju Wang ${ }^{1}$, Feifei Fan', Jiangwei Wang ${ }^{2}$, Haoran Wang ${ }^{3}$, Siyu Tao ${ }^{1}$, Avery Yang ${ }^{1}$, Yang Liu 4 , Huck Beng Chew ${ }^{3}$, Scott X. Mao ${ }^{2}$, Ting Zhu' ${ }^{1} \&$ Shuman Xia ${ }^{1}$

Mechanical degradation and resultant capacity fade in high-capacity electrode materials critically hinder their use in high-performance rechargeable batteries. Despite tremendous efforts devoted to the study of the electro-chemo-mechanical behaviours of high-capacity electrode materials, their fracture properties and mechanisms remain largely unknown. Here we report a nanomechanical study on the damage tolerance of electrochemically lithiated silicon. Our in situ transmission electron microscopy experiments reveal a striking contrast of brittle fracture in pristine silicon versus ductile tensile deformation in fully lithiated silicon. Quantitative fracture toughness measurements by nanoindentation show a rapid brittle-to-ductile transition of fracture as the lithium-to-silicon molar ratio is increased to above 1.5. Molecular dynamics simulations elucidate the mechanistic underpinnings of the brittle-to-ductile transition governed by atomic bonding and lithiation-induced toughening. Our results reveal the high damage tolerance in amorphous lithium-rich silicon alloys and have important implications for the development of durable rechargeable batteries.

\footnotetext{
${ }^{1}$ Woodruff School of Mechanical Engineering, Georgia Institute of Technology, Atlanta, Georgia 30332, USA. ${ }^{2}$ Department of Mechanical Engineering and Materials Science, University of Pittsburgh, Pittsburgh, Pennsylvania 15261, USA. ${ }^{3}$ Department of Aerospace Engineering, University of Illinois at UrbanaChampaign, Urbana, Illinois 61801, USA. ${ }^{4}$ Center for Integrated Nanotechnologies, Sandia National Laboratories, Albuquerque, New Mexico 87185, USA Correspondence and requests for materials should be addressed to T.Z. (email: ting.zhu@me.gatech.edu) or to S.X. (email: shuman.xia@me.gatech.edu).
} 
E nergy storage with high performance and low cost is critical for applications in consumer electronics, zero-emission electric vehicles and stationary power management ${ }^{1,2}$. Lithium-ion batteries (LIBs) are the widely used energy storage systems due to their superior performance ${ }^{3-5}$. The operation of a LIB involves repeated insertion and extraction of lithium (Li) ions in active battery electrodes, which are often accompanied with considerable volume changes and stress generation $^{6-12}$. In the development of next-generation LIBs, mechanical degradation in high-capacity electrode materials arises as a bottleneck. Such high-capacity electrode materials usually experience large volume changes (for example, up to about $280 \%$ for silicon (Si)), leading to high mechanical stresses and fracture of electrodes during electrochemical cycling ${ }^{13-22}$. Fracture causes the loss of active materials and yields more surface areas for solid electrolyte interphase (SEI) growth, both of which contribute to the fast capacity fade of LIBs.

To mitigate the mechanical degradation in LIBs, it is essential to quantitatively understand the electrochemically-induced mechanical responses and fundamental mechanical properties of high-capacity electrode materials. The past decade has witnessed a marked increase in studies on the mechanical behaviours of high-capacity electrode materials ${ }^{6-22}$. Focusing on the promise of $\mathrm{Si}$ as the next-generation anode material, significant progress has been made in the experimental measurement and modelling of lithiation/delithiationinduced stresses ${ }^{11,23}, \mathrm{Li}$ concentration-dependent modulus and hardness ${ }^{24-26}$, time-dependent $\mathrm{creep}^{26,27}$ and strain-rate sensitivities ${ }^{28}$. However, there is still a critical lack of fundamental understanding on the fracture-related properties $^{29,30}$, which ultimately dictate the resistance of the electrode material to mechanical degradation and failure.

Here we report an integrated experimental and computational investigation on the damage tolerance of lithiated $\mathrm{Si}$. We performed in situ nanomechanical bending test on a partially lithiated Si nanowire inside a transmission electron microscope (TEM). The result reveals a direct contrast between the low fracture resistance in the brittle core of pristine $\mathrm{Si}$ and the high damage tolerance in the ductile shell of fully lithiated Si. We conducted systematic measurements of the fracture toughness of lithiated $\mathrm{Si}$ thin films with nanoindentation. The results show a rapid brittle-to-ductile transition of fracture in $\mathrm{Li}_{x} \mathrm{Si}$ alloys with increasing Li concentration. We also performed continuum finite element (FE) and molecular dynamics (MD) simulations to interpret these experimental findings, thereby gaining mechanistic insights into the fracture mechanisms of lithiated Si. These results have important implications for the development of durable Si-based anodes for next-generation LIBs.

\section{Results}

In situ electrochemical and bending tests of $\mathrm{Si}$ nanowires. Figure 1a,b show the schematic illustrations of in situ electrochemical lithiation and fracture testing of a single $\mathrm{Si}$ nanowire inside a TEM. The nano-sized electrochemical cell ${ }^{31}$ consisted of a $\mathrm{Si}$ nanowire on $\mathrm{Si}$ substrate as the working electrode and a $\mathrm{Li}$ probe as the counter electrode; the native $\mathrm{Li}_{2} \mathrm{O}$ layer on $\mathrm{Li}$ surface served as a solid electrolyte. The $<111>$ oriented single crystal Si nanowire had an initial diameter of about $142 \mathrm{~nm}$. To start the lithiation, the Li probe was brought into contact with the free end of the Si nanowire and then a bias voltage of $-2 \mathrm{~V}$ was applied between the working and the counter electrodes. Because Li diffusion/reaction on the surface of $\mathrm{Si}$ is much faster than that in the bulk ${ }^{14}, \mathrm{Li}$ ions first migrated preferably along the free surface of the Si nanowire and then diffused towards its centre. In the bulk of the $\mathrm{Si}$ nanowire, lithiation proceeded in a core-shell mode, yielding a core of crystalline $\mathrm{Si}(c-\mathrm{Si})$ and a shell of amorphous $\mathrm{Li}_{x} \mathrm{Si}\left(a-\mathrm{Li}_{x} \mathrm{Si}\right.$, $x \approx 3.75)$. The core-shell interface was atomically sharp and oriented parallel to the longitudinal axis of the nanowire ${ }^{12}$. Across this interface, an abrupt change of $\mathrm{Li}$ concentration occurred, resulting in high compressive stresses near the core-shell interface (that is, behind the lithiation front) ${ }^{32}$. The lithiation-induced compression slowed down and eventually stalled the movement of the interface. At the end of the lithiation process, the diameter of the unlithiated $c$-Si core was $85 \mathrm{~nm}$; the thicknesses of the $a-\mathrm{Li}_{3.75} \mathrm{Si}$ shell and the layer of lithiated surface oxide $\left(\mathrm{Li}_{y} \mathrm{SiO}_{z}\right)$ were 47 and $17 \mathrm{~nm}$, respectively.

After the lithiation experiment, the partially lithiated $\mathrm{Si}$ nanowire was subjected to in situ compression by the Li probe, as shown in Fig. 1c and Supplementary Movie 1. As the compressive load was increased, the nanowire buckled and subsequently bent, resulting in a single sharp kink in the nanowire. Figure 1d shows a zoom-in TEM image near the kinked region. It is seen that brittle fracture occurred in the unlithiated $c$-Si core, evidenced by the nearly flat fracture surfaces. The fracture strength of the $c$-Si core was determined to be about $7.7 \mathrm{GPa}$, which is consistent with the literature data on the strength of pristine $<111\rangle$-oriented Si nanowires with similar sizes (see Supplementary Fig. 1 and related text). In contrast, the $a-\mathrm{Li}_{3.75} \mathrm{Si}$ shell underwent considerably large tensile deformation accompanied by pronounced lateral thinning,
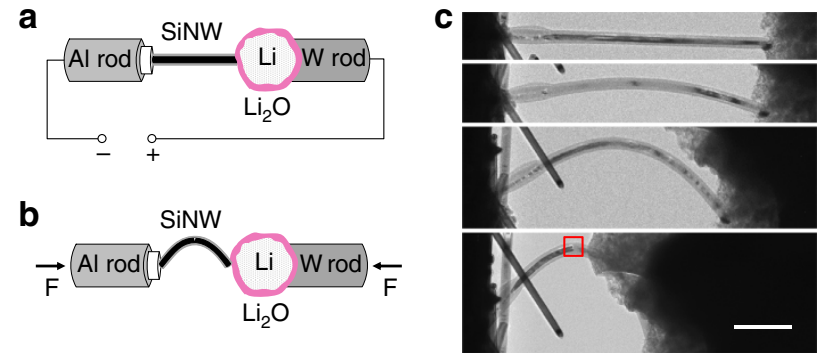

d

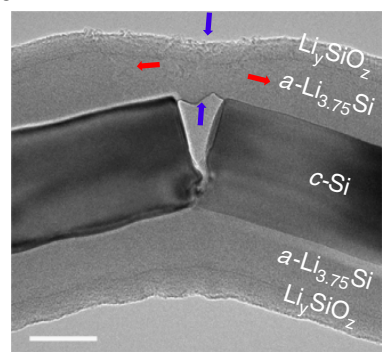

e

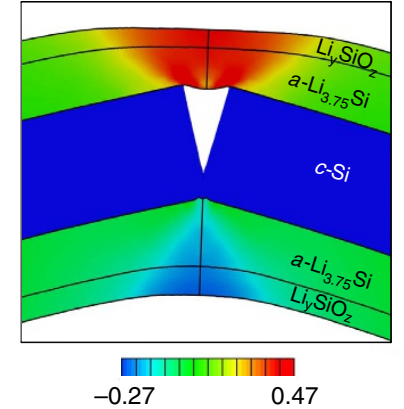

Figure 1 | In situ electrochemical and bending test of a Si nanowire.

(a) Schematic of the in situ TEM setup for a nano-sized electrochemical cell. (b) Schematic of the buckling and bending test of a partially lithiated $\mathrm{Si}$ nanowire subjected to the compressive force F. (c) Sequential TEM images showing the process of axial compression; bucking and bending of the partially lithiated Si nanowire gave rise to a sharply kinked region indicated by the red box (scale bar, $1 \mu \mathrm{m}$ ). (d) Zoom-in TEM image (that is, the red box region in c) showing the brittle fracture of the unlithiated c-Si core, as well as the large tensile deformation (red arrows) and lateral thinning (blue arrows) of the lithiated $a-\mathrm{Li}_{3.75} \mathrm{Si}$ shell (scale bar, $50 \mathrm{~nm}$ ). (e) Finite element result showing the simulated elastic-plastic deformation in the nanowire that agrees with the TEM image in $\mathbf{d}$. Colour contour reveals the distribution of axial strain in the lithiated $a-\mathrm{Li}_{3.75} \mathrm{Si}$ shell, with an extraordinarily large tensile strain of about $47 \%$ occurring in the freestanding part of $a-\mathrm{Li}_{3.75} \mathrm{Si}$. 
without observable damages such as cracking or shear banding. The lithiated surface oxide layer $\left(\mathrm{Li}_{y} \mathrm{SiO}_{z}\right)$ remained coherent to the $a-\mathrm{Li}_{3.75} \mathrm{Si}$ shell and thus underwent similar straining as the latter. Figure 1e shows the result of a continuum FE simulation of large local deformation near the kinked region (see the modelling details in Supplementary Fig. 4 and Supplementary Methods). In the FE simulation, we assumed that the brittle fracture of the $c$-Si core was a much faster process than the elastic-plastic deformation in the $a-\mathrm{Li}_{3.75} \mathrm{Si}$ shell, such that the latter occurred after the crack in the $c$-Si core had been fully formed. As a result, the large tensile deformation in the $a-\mathrm{Li}_{3.75} \mathrm{Si}$ shell was primarily accommodated by the sliding at the core-shell interface, yielding a large crack opening in the $c$-Si core near the core-shell interface. The deformation morphology from the FE simulation (Fig. 1e) was in close agreement with that in the TEM image (Fig. 1d). The most striking observation from Fig. 1d,e is the large tensile (plastic) strain of $\sim 47 \%$ occurring in the $a-\mathrm{Li}_{3.75} \mathrm{Si}$ shell, with a concurrent large lateral contraction (that is, thinning) of $\sim 45 \%$. Therefore, the in situ TEM experiment and FE simulation demonstrate that the amorphous Li-rich $\mathrm{Si}$ alloy $\left(a-\mathrm{Li}_{3.75} \mathrm{Si}\right)$ exhibits extraordinary damage tolerance, while $c$-Si is brittle as expected.

Fracture toughness measurements of lithiated Si. To quantitatively study the damage tolerance of electrochemically lithiated $\mathrm{Si}$ at different $\mathrm{Li}$ concentrations, we employed an in-house developed nanoindentation system (Supplementary Fig. 2) to characterize the fracture toughness of $a-\mathrm{Li}_{x} \mathrm{Si}$, a property that describes the ability of a material to resist crack propagation ${ }^{33}$. The Si electrodes for nanoindentation tests were fabricated in a thin-film form by sputter deposition of $325 \mathrm{~nm}$ thick amorphous $\mathrm{Si}(a-\mathrm{Si})$ films onto flat titanium (Ti) substrates. Each Si electrode was lithiated in a custom-fabricated Teflon electrochemical cell, as illustrated in Fig. 2a, with a Li foil as the reference electrode. During the electrochemical testing of each $a$-Si electrode, a Michelson interferometer was used to measure the curvature change of the substrate from which the lithiation-induced biaxial film stress was deduced using Stoney's equation ${ }^{34}$.

Figure $2 \mathrm{~b}, \mathrm{c}$ show the electrochemical profiles and corresponding film stresses measured for five $a$-Si thin-film electrodes. The $\mathrm{Li}$ concentrations in these electrodes were controlled by galvanostatical lithiation at $20 \mu \mathrm{A} \mathrm{cm}^{-2}$ to different cutoff potentials. The sloping voltage profiles below $0.5 \mathrm{~V}$ versus $\mathrm{Li} / \mathrm{Li}^{+}$ suggest the formation of a single-phase $a-\mathrm{Li}_{x} \mathrm{Si}$ during lithiation. As shown in Fig. 2c, all thin-film electrodes exhibited an initial compressive stress of about $200 \mathrm{MPa}$ that resulted from the sputtering process. The compressive stress in the thin-film electrodes increased to $500-800 \mathrm{MPa}$ at the end of lithiation, due to the substrate constraint on lithiation-induced volume expansion. This large residual compressive stress may impede crack growth during nanoindentation testing and thus affect the measurement of fracture toughness. To reduce the influence of those residual stresses, we first lithiated the $a$-Si electrode to a targeted $\mathrm{Li}$ concentration and then delithiated it to $0.25-0.3 \mathrm{~V}$ above the lithiation cutoff potential. During the delithiation, a small fraction of $\mathrm{Li}$ was extracted from the electrodes, resulting in small volume shrinkages so as to reverse the film stress from compression to tension. The tensile stress in the thin film was small, but found to be beneficial for promoting the nanoindentation-induced cracking and enabling the evaluation of fracture toughness of $a-\mathrm{Li}_{x} \mathrm{Si}$. The effects of SEI formation on electrode cracking were estimated to be negligibly small ${ }^{35}$ and therefore were not considered in the fracture toughness measurement.

To measure the facture toughness, the $a-\mathrm{Li}_{x} \mathrm{Si}$ thin-film electrodes were indented with a cube-corner indenter tip in an
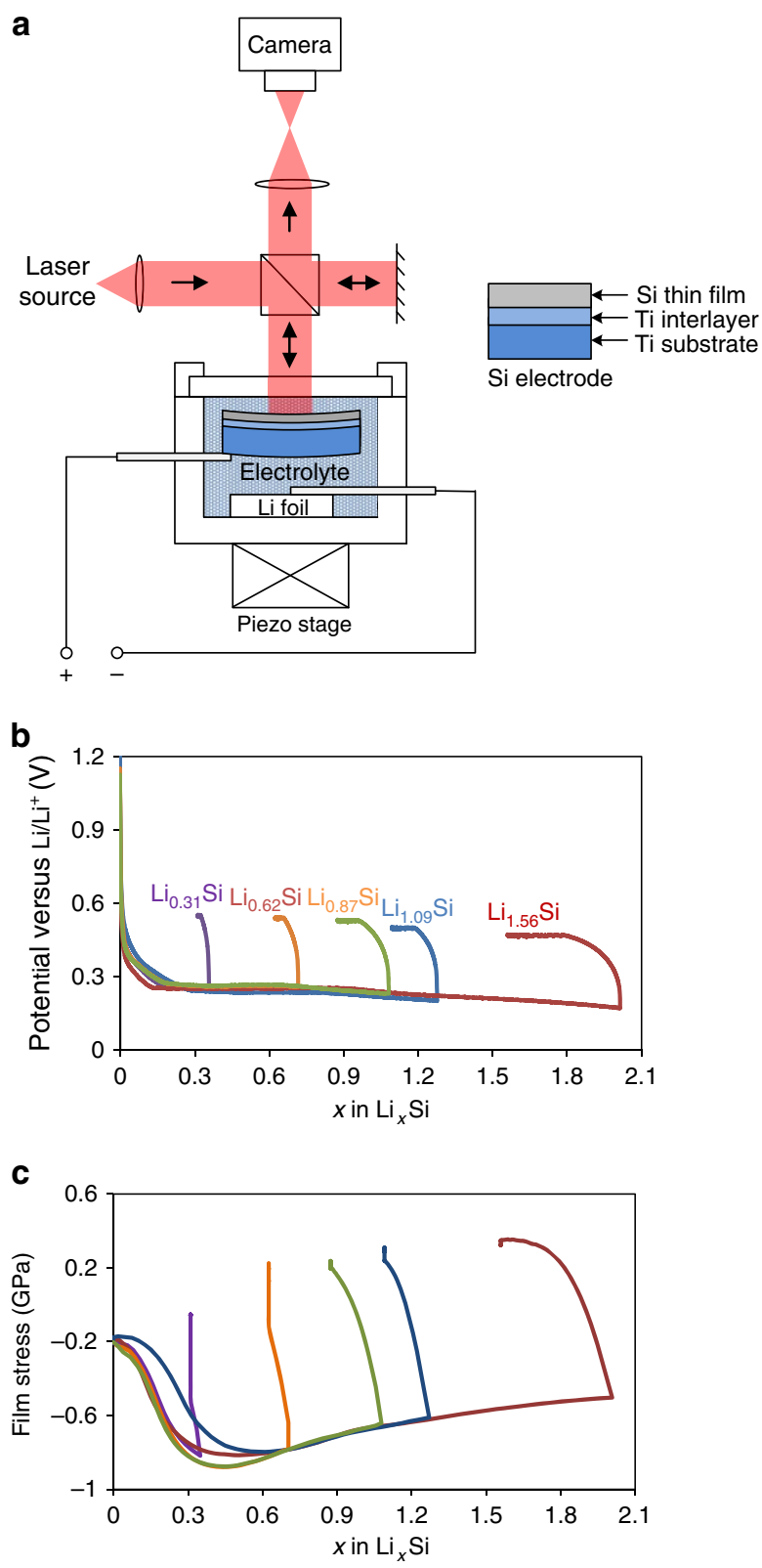

Figure 2 | Electro-chemo-mechanical characterization of Si electrodes.

(a) Schematic illustration of an electrochemical cell, consisting of a Si thinfilm working electrode, a liquid electrolyte, and a Li foil counter electrode, as well as a Michelson interferometer setup for in situ film stress measurement. (b) Electrochemical profiles of five $325 \mathrm{~nm}$ Si electrodes that were galvanostatically lithiated and delithiated to various Li concentrations, followed by potentiostatic delithiation. (c) Evolution of the film stress in the five Si electrodes corresponding to the electrochemical profiles in $\mathbf{b}$.

argon-filled glove box. After unloading of the indenter, residual indents and surrounding areas were imaged using a scanning electron microscope (SEM). Figure 3a-c show the nanoindentation results for a partially lithiated $\mathrm{Si}$ electrode with low $\mathrm{Li}$ concentration $\left(\mathrm{Li}_{0.87} \mathrm{Si}\right)$ under different indentation loads, which exhibit distinct cracking behaviours including (i) no cracking, (ii) indent corner cracking and (iii) massive cracking. Specifically, under a small load of $3.92 \mathrm{mN}$, residual plastic deformation at the permanent indent was observed without obvious cracking (Fig. 3a). As the load was increased to $9.8 \mathrm{mN}$, three radial cracks emanated from the sharp corners of the indent (Fig. 3b). A further increase in the indentation load to $29.4 \mathrm{mN}$ resulted in 

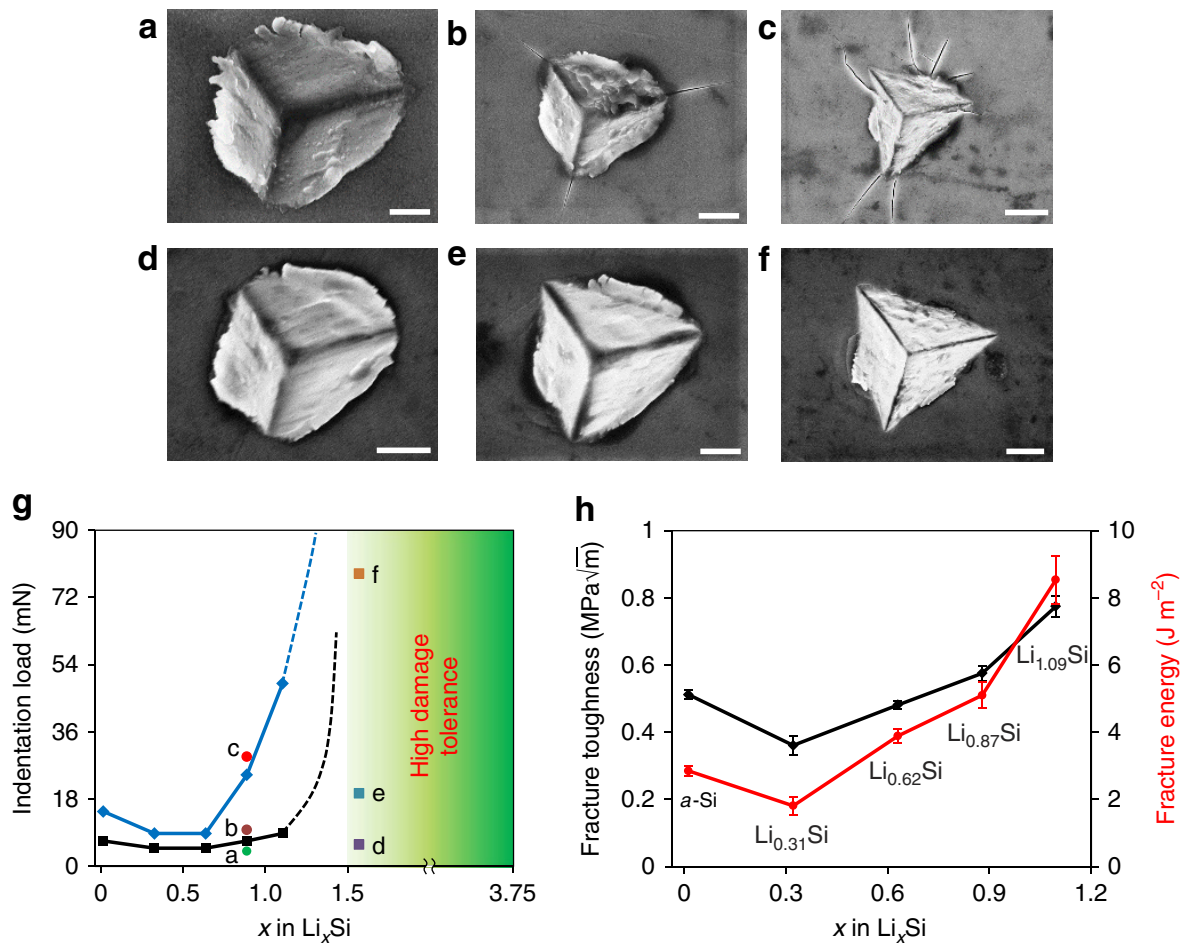

Figure 3 | Fracture toughness measurement by nanoindentation. (a-c) SEM images of residual indents for a lithiated electrode of $\mathrm{Li}_{0.87} \mathrm{Si}$, showing (a) no cracking, (b) radial cracking and (c) massive cracking subjected to different applied indentation loads (scale bar, $0.5 \mu \mathrm{m}$ in $\mathbf{a} ; 1 \mu \mathrm{m}$ in $\mathbf{b} ; 2 \mu \mathrm{m}$ in $\mathbf{c}$ ). (d-f) SEM images of residual indents for a lithiated $\mathrm{Si}$ electrode of $\mathrm{Li}_{1.56} \mathrm{Si}$ showing no cracking subjected to different applied indentation loads (scale bar, $1 \mu \mathrm{m}$ in $\mathbf{d}$ and $\mathbf{e} ; 2 \mu \mathrm{m}$ in $\mathbf{f}$ ). (g) The indentation loads (symbols) applied to the lithiated electrodes with different Li contents, corresponding to images in (a-f). The blue solid curve represents the upper load limit above which massive cracking occurred and the black solid curve the lower limit below which no crack was induced. The dashed lines show the qualitative trends interpolated from the data. (h) Fracture toughness and fracture energy of lithiated $\mathrm{Si}$ as a function of Li concentration.

massive cracking around the indent (Fig. 3c). Similar cracking behaviours were observed for low Li concentrations from $x=0$ to 1.09. However, as the Li:Si ratio was increased to above 1.56, no cracking was observed from indents for a wide range of indentation loads up to $93 \mathrm{mN}$, as shown in Fig. 3d-f. Critical indentation loads separating the above three regimes of cracking behaviours are plotted in Fig. 3g. The two critical load curves, respectively, represent the upper load limit above which massive cracking occurred and the lower limit below which no crack was induced. The upper load limit curve varies substantially with the Li concentration when $x$ exceeds 0.6 , indicating that the fracture toughness of lithiated $\mathrm{Si}$ starts to depend sensitively on the $\mathrm{Li}$ concentration. Similar drastic change of the lower load limit curve can be inferred as $x$ varies from 1.09 to 1.56 (as indicated by the dashed line), since no cracking was observed for indentation loads up to $93 \mathrm{mN}$ when $x=1.56$. Therefore, our results indicate that a brittle-to-ductile transition of fracture occurs when the $\mathrm{Li}$ composition falls in the range of $x=1.09-1.56$. For $x \leq 1.09$, the indentation loads in between the two limits induced welldeveloped radial cracks. We used these loads to evaluate the fracture toughness of $a-\mathrm{Li}_{x} \mathrm{Si}$ with the Morris nanoindentation model (see Supplementary Fig. 3 and Supplementary Methods for model details).

Figure $3 \mathrm{~h}$ shows the measured fracture toughness, $K_{\mathrm{Ic}}$, as a function of $\mathrm{Li}$ concentration. Also shown in this figure is the fracture energy, defined as $\Gamma=K_{\mathrm{Ic}}^{2} / E$, where $E$ is Young's modulus of lithiated Si taken from the experimental measurement ${ }^{25}$. Figure $3 \mathrm{~h}$ clearly reveals that the fracture toughness of $a-\mathrm{Li}_{x} \mathrm{Si}$ alloys depends sensitively on the $\mathrm{Li}$ concentration. For example, the fracture toughness and fracture energy of unlithiated $a$-Si are $0.51 \pm 0.014 \mathrm{MPa} \sqrt{\mathrm{m}}$ and $2.85 \pm 0.15 \mathrm{~J} \mathrm{~m}^{-2}$, respectively. These low values are typical of brittle materials with little fracture resistance ${ }^{33}$. As the Li concentration increases, the fracture resistance of lithiated Si first decreases slightly, indicating lithiation-induced embrittlement. This trend is in qualitative agreement with the recent $a b$ initio calculations ${ }^{36}$ that show a small amount of $\mathrm{Li}$ insertion into $\mathrm{Si}$ substantially weakens $\mathrm{Si}-\mathrm{Si}$ bonds, and hence reduces the surface energy of the material. However, upon further lithiation beyond $x=0.31$, both the measured fracture toughness and fracture energy increase substantially with increasing $\mathrm{Li}$ concentration, reaching $0.77 \pm 0.03 \mathrm{MPa} \sqrt{\mathrm{m}}$ and $8.54 \pm 0.72 \mathrm{~J} \mathrm{~m}^{-2}$ for $\mathrm{Li}_{1.09} \mathrm{Si}$, respectively. Since a further increase in Li concentration gave rise to large residual plastic deformation at and around indents without cracking (for example, Fig. 3d-f), we take the Li:Si ratio of $x \approx 1.5$ as the characteristic composition above which the brittle-to-ductile transition of fracture occurs in $a-\mathrm{Li}_{x} \mathrm{Si}$ alloys.

Atomistic modelling of fracture in lithiated Si. To understand the experimentally measured brittle-to-ductile transition phenomenon, we performed MD simulations of deformation and fracture in $a-\mathrm{Li}_{x} \mathrm{Si}$ alloys using the reactive force field ${ }^{23}$ (see Methods section). In Fig. 4, we contrast the MD results by showing the brittle crack growth in Li-lean $a$ - $\mathrm{Li}_{0.5} \mathrm{Si}$ versus the ductile crack blunting in Li-rich $a-\mathrm{Li}_{2.5} \mathrm{Si}$. In both cases, MD simulations were performed for samples containing a sharp-edge pre-crack and subjected to a far-field tensile load. Figure 4a presents a sequence of MD snapshots showing brittle crack growth in $a-\mathrm{Li}_{0.5} \mathrm{Si}$. In this case, the local tensile deformation near the crack tip is primarily accommodated by the stretching and 


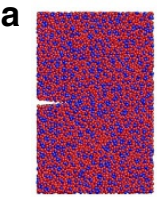

$\varepsilon=0$

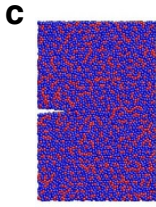

$\varepsilon=0$

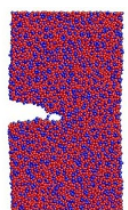

$10.55 \%$

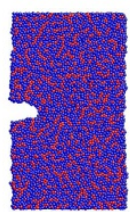

$10.44 \%$

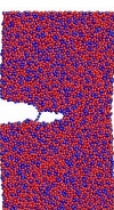

$10.70 \%$

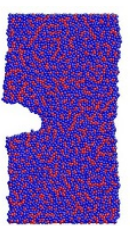

$17.57 \%$
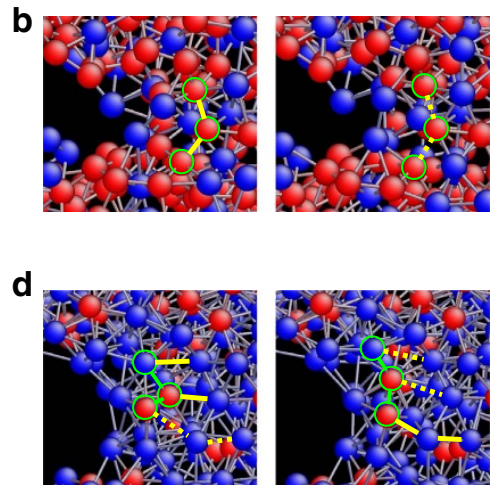

e

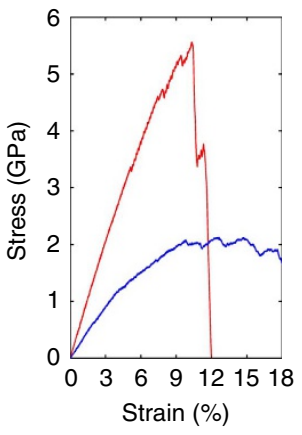

Figure 4 I Molecular dynamics (MD) simulations. (a) MD snapshots of brittle fracture in Li-lean $\mathrm{Si}$ (a-Li ${ }_{0.5} \mathrm{Si}$ ) at various stages of applied strain load $\varepsilon$, showing the growth of an atomically sharp crack. (b) Zoom-in images near the crack tip in strained $a$-Lio.5 $\mathrm{Si}$, showing the characteristic atomic processes of Si-Si bond breaking (from solid to dashed lines). (c) MD snapshots of ductile response in Li-rich $\mathrm{Si}\left(a\right.$-Li ${ }_{2.5} \mathrm{Si}$ ) at various stages of applied strain

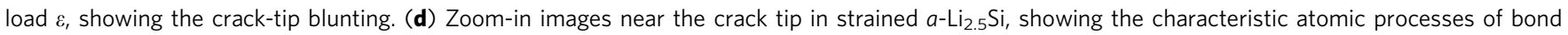
breaking (from solid to dashed lines), formation (from dashed to solid lines) and rotation (angle change between two green lines). Si atoms are coloured by red and $\mathrm{Li}$ by blue in a-d. (e) Overall stress-strain responses of $a$ - $\mathrm{Li}_{0.5} \mathrm{Si}$ (red curve) and $a$ - $\mathrm{Li}_{2.5} \mathrm{Si}$ (blue curve).

breaking of $\mathrm{Si}-\mathrm{Si}$ bonds, as shown in Fig. $4 \mathrm{~b}$. Due to the high fraction of strong covalent $\mathrm{Si}$ bonds, the bond alteration events are mostly discrete and disruptive, lacking bond reformation. A small damage zone forms near the crack tip, and it contains a high fraction of dangling bonds and atomic-sized voids. As the crack extends through such a damage zone, the crack faces become atomically rough, but the crack tip remains sharp, as seen from Fig. 4a. In contrast, Fig. 4c presents a sequence of MD snapshots showing the crack blunting in $a-\mathrm{Li}_{2.5} \mathrm{Si}$. In this case, the local tensile deformation near the crack tip is accommodated by the stretching, rotation, breaking and frequent reformation of atomic bonds, as shown in Fig. $4 \mathrm{~d}$. Several Li atoms often collectively participate in a bond-switching process, so that the amorphous structure near the crack tip remains nearly homogenous without significant damage. As a result, the crack tip becomes considerably blunt without apparent crack growth, as seen from Fig. 4c. In addition, we compare the stress-strain curves during tensile straining of the above two pre-cracked samples in Fig. 4e; the fast brittle fracture in $a-\mathrm{Li}_{0.5} \mathrm{Si}$ is manifested as a sharp load drop, while the ductile crack blunting in $a-\mathrm{Li}_{2.5} \mathrm{Si}$ is characterized by a gradual load decrease, indicative of extensive plastic deformation near the crack tip. The above MD results of brittle versus ductile behaviours in Li-lean and $\mathrm{Li}$-rich $a-\mathrm{Li}_{x} \mathrm{Si}$ alloys are consistent with the trend in nanoindentation measurements. Hence, our MD study reveals a plausible atomistic mechanism of brittle-to-ductile transition in $a-\mathrm{Li}_{x} \mathrm{Si}$ alloys, namely, the decreasing fraction of strong covalent $\mathrm{Si}$ bonding and the concomitant increasing fraction of delocalized metallic $\mathrm{Li}$ bonding give rise to an alteration of the dominant atomic-level processes of deformation and fracture with increasing Li concentration.

\section{Discussion}

The above experimental and modelling results underscore the notion of the high damage tolerance of amorphous Li-rich $\mathrm{Si}$ alloys and thus have important implications for the design of durable Si-based anodes for next-generation LIBs. Recently, lithiation experiments have been conducted with $a$-Si nanoparticles with a wide range of diameters up to $870 \mathrm{~nm}^{18}$, as well as with $a$-Si pillars of a few microns in diameter ${ }^{37}$. In those experiments, no cracking and fracture were observed in lithiated $a$-Si electrodes. However, it has been shown that the surface layers of lithiated $a$-Si particles and wires should have undergone large hoop tensile deformation resulting from the two-phase lithiation and associated volume expansion at curved phase boundaries ${ }^{17,18}$. Given the large hoop tension and accordingly high driving force of fracture, the lack of observed cracking ${ }^{37,38}$ implies that the fully lithiated $a$-Si should be mechanically robust. In this work, our in situ TEM and nanoindentation experiments collectively provide direct evidence for the high damage tolerance (that is, high tensile ductility and high fracture toughness) of Li-rich Si alloys, which is the essential mechanistic information for the design of durable Si-based electrodes. In addition, the quantitative fracture characteristics obtained in our work constitute an important input for optimizing the microstructures and lithiation/delithiation windows of Si-based LIBs. Incidentally, the mechanical robustness of lithiated $a-\mathrm{Si}$ is in contrast with the commonly observed surface cracking and fracture after lithiation in large $c$-Si nanostructures ${ }^{16,19}$. The ease of fracture in lithiated $c$-Si has been attributed to a crystallographic effect on the lithiation anisotropy, resulting in inhomogeneous phases and deformation near the interface between anisotropically lithiated domains ${ }^{39}$. For $a-S i$ electrodes, there is no such lithiation anisotropy. As a result, both the lithiated phases and deformation processes in $a$-Si particles and wires are more homogenous than those in $c$-Si counterparts, which contribute to the high mechanical robustness of the former.

To conclude, we have integrated experiments and modelling to reveal the high damage tolerance of electrochemically lithiated $\mathrm{Si}$ electrodes. The in situ TEM experiment on a partially lithiated Si nanowire showed a striking contrast of the brittle fracture in the unlithiated $\mathrm{Si}$ core versus the ductile tensile deformation in the lithiated Si shell. The nanoindentation testing of amorphous lithiated Si alloys indicated a drastic increase of fracture toughness as the $\mathrm{Li}$ to $\mathrm{Si}$ ratio was increased to above 1.5. Our atomistic simulations elucidated the mechanistic underpinnings of the brittle-to-ductile transition in terms of atomic bonding and lithiation-induced toughening. The quantitative characterization and mechanistic understanding of high damage tolerance of Li-rich $\mathrm{Si}$ alloys represent a critical step towards the rational design of durable Si-based electrodes for next-generation LIBs. Broadly, our integrated experimental and modelling approach can be applied to the mechanical characterization of a wide range of electrochemically active materials for energy storage applications. 


\section{Methods}

Thin-film electrode fabrication and electrochemical cell assembly. Single-side polished titanium (Ti) plates with a thickness of $0.5 \mathrm{~mm}$ were used as substrates for fabrication of thin-film electrodes, and they also served as current collectors for electrochemical measurements. Before a Ti substrate was placed inside the deposition system (Denton Discovery RF/DC Sputterer), it was etched with 5\% $\mathrm{HCl}$ to remove the surface Ti oxide. A $26 \mathrm{~nm}$ Ti thin film was first sputtered onto the polished side of the Ti substrate, followed by the deposition of a $325 \mathrm{~nm}$ thick $\mathrm{Si}$ film. The Ti interlayer, serving as an adhesion-promoting layer, was prepared by DC sputtering of a Ti target (3" diameter disc, $99.995 \% \mathrm{Ti}$, Kurt J. Lesker Co., Livermore, CA) at a power of $53 \mathrm{~W}$ and a pressure of $6.29 \mathrm{mTorr}$ of argon. The $\mathrm{Si}$ film was prepared by RF magnetron sputtering of an Si target (3" diameter disc, 99.995\% Si, Kurt J. Lesker Co., Livermore, CA, USA) at a power of $200 \mathrm{~W}$ and a pressure of 5 mTorr of argon. Previous studies have shown that $\mathrm{Si}$ thin films formed under these sputtering conditions are amorphous ${ }^{40}$

After $\mathrm{Si}$ was deposited on the polished side of the Ti substrate, a thin layer of polydimethylsiloxane (PDMS) was coated on the unpolished side of the Ti substrate to prevent the formation of an SEI layer on the Ti surface. The Si film-coated Ti electrode was then assembled into a custom-fabricated Teflon electrochemical cell with a glass window (Fig. 2a) inside an argon-filled glove box that was maintained at $<0.1 \mathrm{ppm}$ of $\mathrm{O}_{2}$ and $\mathrm{H}_{2} \mathrm{O}$. A Li foil was used as the reference/counter electrode, and $1 \mathrm{M}$ of lithium hexafluoro-phosphate $\left(\mathrm{LiPF}_{6}\right)$ in 1:1:1 (weight \%) ratio of ethylene carbonate $(\mathrm{EC})$ to dimethyl carbonate (DMC) to diethyl carbonate (DEC) was used as the electrolyte.

Electrochemical and in situ film stress measurement. The electrochemical measurement was conducted with a battery tester (UBA 5, Vencon Technologies, Ontario, Canada). Five Si electrode samples were first lithiated galvanostatically at $20 \mu \mathrm{A} \mathrm{cm}^{-2}$ with pre-determined cutoff potentials. The lithiation was followed by delithation at the same current density until potentials of $0.2-0.3 \mathrm{~V}$ versus $\mathrm{Li} / \mathrm{Li}{ }^{+}$ above the cutoff potentials for lithiation were reached. Subsequently, delithiation was continued potentiostatically until the current was reduced below $0.2 \mu \mathrm{Acm}^{-2}$. The Si electrodes were then removed from the cell inside the glove box and thoroughly cleaned by rinsing them with DMC. Stress evolution in the Si films during lithiation and delithiation was measured by monitoring the substrate curvature with a Michelson interferometer. The biaxial film stress was deduced from the measured curvature using Stoney's equation ${ }^{34}$.

Nanoindentation experiments. The fracture toughness of lithiated Si electrodes was measured by an in-house developed nanoindentation system inside an argonfilled glove box (Supplementary Fig. 2). Peak loads, ranging from 1 to $93 \mathrm{mN}$ at constant loading and unloading rates of $500 \mu \mathrm{N} \mathrm{s}^{-1}$, were used during nanoindentation tests. Ten indents at each load were made for each Si electrode sample and spaced $100 \mu \mathrm{m}$ apart. The indented Si electrodes were imaged by a Zeiss Ultra60 FESEM (Carl Zeiss Microscopy, LLC, North America, Peabody, MA) operated at an accelerating voltage of $5 \mathrm{kV}$. During the sample transfer from the glove box to the SEM chamber, the lithiated Si electrodes were coated with a thin layer of anhydrous DMC to avoid the reaction of lithiated Si with ambient oxygen and moisture.

Molecular dynamics simulations. Molecular dynamics simulations were performed using the large-scale atomic molecular massively parallel simulator (LAMMPS). Atomic interactions were modelled by a reactive force field developed for $\mathrm{Li}-\mathrm{Si}$ alloys ${ }^{23}$. The $a-\mathrm{Li}_{x} \mathrm{Si}$ samples were prepared from melting-and-quenching simulations with a quench rate of $2 \times 10^{12} \mathrm{Ks}^{-1}$. In these simulations, the initial structures were created by randomly inserting $\mathrm{Li}$ atoms into a crystalline $\mathrm{Si}$ lattice. After quenching at zero stresses, the $a-\mathrm{Li}_{x} \mathrm{Si}$ samples had the final dimensions of $\sim 16 \times 10 \times 1.5 \mathrm{~nm}$. A sharp crack with a length of $\sim 3 \mathrm{~nm}$ was created in each sample. The pre-cracked samples were then loaded in tension with a strain rate of $5 \times 10^{8} \mathrm{~s}^{-1}$, while the system temperature was maintained at $5 \mathrm{~K}$. Periodic boundary conditions were applied along the loading and the thickness directions. A plane-strain condition was imposed by fixing the thickness of the simulation box. The time step in all the molecular dynamics simulations was $1 \mathrm{fs}$.

\section{References}

1. Tarascon, J. M. \& Armand, M. Issues and challenges facing rechargeable lithium batteries. Nature 414, 359-367 (2001).

2. Dunn, B., Kamath, H. \& Tarascon, J.-M. Electrical energy storage for the grid: a battery of choices. Science 334, 928-935 (2011).

3. Goodenough, J. B. \& Kim, Y. Challenges for Rechargeable Li Batteries. Chem. Mater. 22, 587-603 (2010).

4. Magasinski, A. et al. High-performance lithium-ion anodes using a hierarchical bottom-up approach. Nat. Mater. 9, 353-358 (2010).

5. Li, Y. et al. Current-induced transition from particle-by-particle to concurrent intercalation in phase-separating battery electrodes. Nat. Mater. 13, 1149-1156 (2014).

6. Huggins, R. A. \& Nix, W. D. Decrepitation model for capacity loss during cycling of alloys in rechargeable electrochemical systems. Ionics 6, 57-63 (2000).
7. Beaulieu, L. Y., Eberman, K. W., Turner, R. L., Krause, L. J. \& Dahn, J. R. Colossal reversible volume changes in lithium alloys. Electrochem. Solid State Lett. 4, A137-A140 (2001).

8. Chan, C. K. et al. High-performance lithium battery anodes using silicon nanowires. Nat. Nanotech. 3, 31-35 (2008).

9. Cheng, Y. T. \& Verbrugge, M. W. Evolution of stress within a spherical insertion electrode particle under potentiostatic and galvanostatic operation. J. Power Sources 190, 453-460 (2009).

10. Zhao, K., Pharr, M., Vlassak, J. J. \& Suo, Z. Fracture of electrodes in lithium-ion batteries caused by fast charging. J. Appl. Phys. 108, 073517 (2010).

11. Sethuraman, V. A., Chon, M. J., Shimshak, M., Srinivasan, V. \& Guduru, P. R. In situ measurements of stress evolution in silicon thin films during electrochemical lithiation and delithiation. J. Power Sources 195, 5062-5066 (2010).

12. Liu, X. H. et al. In situ atomic-scale imaging of electrochemical lithiation in silicon. Nat. Nanotech. 7, 749-756 (2012).

13. Li, H., Huang, X. J., Chen, L. Q., Wu, Z. G. \& Liang, Y. A high capacity nano-Si composite anode material for lithium rechargeable batteries. Electrochem. Solid State Lett. 2, 547-549 (1999).

14. Liu, X. H. et al. Anisotropic Swelling and Fracture of Silicon Nanowires during Lithiation. Nano Lett. 11, 3312-3318 (2011).

15. Chon, M. J., Sethuraman, V. A., McCormick, A., Srinivasan, V. \& Guduru, P. R. Real-time measurement of stress and damage evolution during initial lithiation of crystalline silicon. Phys. Rev. Lett. 107, 045503 (2011).

16. Liu, X. H. et al. Size-dependent fracture of silicon nanoparticles during lithiation. ACS Nano 6, 1522-1531 (2012).

17. Wang, J. W. et al. Two-phase electrochemical lithiation in amorphous silicon. Nano Letters 13, 709-715 (2013).

18. McDowell, M. T. et al. In situ TEM of two-phase lithiation of amorphous silicon nanospheres. Nano Lett. 13, 758-764 (2013).

19. McDowell, M. T., Lee, S. W., Nix, W. D. \& Cui, Y. 25th anniversary article: understanding the lithiation of silicon and other alloying anodes for lithium-ion batteries. Adv. Mater. 25, 4966-4984 (2013).

20. Woodford, W. H., Chiang, Y. M. \& Carter, W. C. "Electrochemical Shock" of intercalation electrodes: a fracture mechanics analysis. J. Electrochem. Soc. 157, A1052-A1059 (2010)

21. Haftbaradaran, H., Xiao, X. C., Verbrugge, M. W. \& Gao, H. J. Method to deduce the critical size for interfacial delamination of patterned electrode structures and application to lithiation of thin-film silicon islands. J. Power Sources 206, 357-366 (2012).

22. Wang, H., Hou, B., Wang, X., Xia, S. \& Chew, H. B. Atomic-scale mechanisms of sliding along an interdiffused Li-Si-Cu interface. Nano Lett. 15, 1716-1721 (2015).

23. Fan, F. et al. Mechanical properties of amorphous Li x Si alloys: a reactive force field study. Model. Simul. Mater. Sci. Eng. 21, 074002 (2013).

24. Shenoy, V. B., Johari, P. \& Qi, Y. Elastic softening of amorphous and crystalline Li-Si Phases with increasing Li concentration: a first-principles study. J. Power Sources 195, 6825-6830 (2010).

25. Hertzberg, B., Benson, J. \& Yushin, G. Ex-situ depth-sensing indentation measurements of electrochemically produced Si-Li alloy films. Electrochem. Commun. 13, 818-821 (2011).

26. Berla, L. A., Lee, S. W., Cui, Y. \& Nix, W. D. Mechanical behavior of electrochemically lithiated silicon. J. Power Sources 273, 41-51 (2015).

27. Boles, S. T., Thompson, C. V., Kraft, O. \& Moenig, R. In situ tensile and creep testing of lithiated silicon nanowires. Appl. Phys. Lett. 103, 263906 (2013).

28. Pharr, M., Suo, Z. \& Vlassak, J. J. Variation of stress with charging rate due to strain-rate sensitivity of silicon electrodes of Li-ion batteries. J. Power Sources 270, 569-575 (2014)

29. Kushima, A., Huang, J. Y. \& Li, J. Quantitative fracture strength and plasticity measurements of lithiated silicon nanowires by in situ TEM tensile experiments. ACS Nano 6, 9425-9432 (2012).

30. Pharr, M., Suo, Z. \& Vlassak, J. J. Measurements of the fracture energy of lithiated silicon electrodes of Li-ion batteries. Nano Lett. 13, 5570-5577 (2013).

31. Huang, J. Y. et al. In situ observation of the electrochemical lithiation of a single SnO2 nanowire electrode. Science 330, 1515-1520 (2010).

32. Huang, S., Fan, F., Li, J., Zhang, S. L. \& Zhu, T. Stress generation during lithiation of high-capacity electrode particles in lithium ion batteries. Acta Materialia 61, 4354-4364 (2013).

33. Cook, R. F. Strength and sharp contact fracture of silicon. J. Mater. Sci. 41, 841-872 (2006).

34. Stoney, G. G. The tension of metallic films deposited by electrolysis. Proc. $R$ Soc. Lond. A 82, 172-175 (1909).

35. Nadimpalli, S. P. V. et al. On plastic deformation and fracture in Si films during electrochemical lithiation/delithiation cycling. J. Electrochem. Soc. 160, A1885-A1893 (2013).

36. Chou, C.-Y. \& Hwang, G. S. Surface effects on the structure and lithium behavior in lithiated silicon: a first principles study. Surf. Sci. 612, 16-23 (2013).

37. Berla, L. A., Lee, S. W., Ryu, I., Cui, Y. \& Nix, W. D. Robustness of amorphous silicon during the initial lithiation/delithiation cycle. J. Power Sources 258, 253-259 (2014). 
38. McDowell, M. T. et al. Studying the kinetics of crystalline silicon nanoparticle lithiation with in situ transmission electron microscopy. Adv. Mater. 24, 6034-6041 (2012).

39. Liang, W. et al. Tough germanium nanoparticles under electrochemical cycling. ACS Nano 7, 3427-3433 (2013).

40. Sethuraman, V. A., Kowolik, K. \& Srinivasan, V. Increased cycling efficiency and rate capability of copper-coated silicon anodes in lithium-ion batteries. J. Power Sources 196, 393-398 (2011).

\section{Acknowledgements}

We acknowledge the support of the following grants: NSF-CMMI-1300458 (S.X.), NSF-CMMI-1300805 (H.B.C.), NSF-CMMI-1100205 (T.Z.), NSF-DMR-1410936 (T.Z.), and NSF-CMMI-08010934 through the University of Pittsburgh and Sandia National Lab (S.X.M.). We thank Sergiy Krylyuk and Albert V. Davydov at NIST for providing Si nanowires used for in situ TEM experiments. This work was performed, in part, at the Center for Integrated Nanotechnologies, a US Department of Energy, Office of Basic Energy Sciences user facility. Sandia National Laboratories is a multiprogram laboratory managed and operated by Sandia Corporation, a wholly owned subsidiary of Lockheed Martin Corporation, for the US Department of Energy's National Nuclear Security Administration under Contract DE-AC04-94AL85000.

\section{Author contributions}

S.X. conceived the idea, designed and supervised the electrochemical lithiation and nanoindentation experiments. X.W. and A.Y. conducted the electrochemical lithiation and nanoindentation experiments. Y.L., J.W. and S.X. conducted the TEM study. T.Z. and F.F. conducted the MD study. T.Z. and S.T. conducted the FE study of nanowire bending. H.C. and H.W. performed the FE study of nanoindentation. S.X., X.W. and T.Z. wrote the manuscript. All authors discussed and commented on the manuscript.

\section{Additional information}

Supplementary Information accompanies this paper at http://www.nature.com/ naturecommunications

Competing financial interests: The authors declare no competing financial interests.

Reprints and permission information is available online at http://npg.nature.com/ reprintsandpermissions/

How to cite this article: Wang, X. et al. High damage tolerance of electrochemically lithiated silicon. Nat. Commun. 6:8417 doi: 10.1038/ncomms9417 (2015).

\section{(c) (i)}

This work is licensed under a Creative Commons Attribution 4.0 International License. The images or other third party material in this article are included in the article's Creative Commons license, unless indicated otherwise in the credit line; if the material is not included under the Creative Commons license, users will need to obtain permission from the license holder to reproduce the material. To view a copy of this license, visit http://creativecommons.org/licenses/by/4.0/ 